\title{
Role of respiratory sleep disorders in the pathogenesis of nocturnal angina and arrhythmias
}

\author{
R. Liston, P.C. Deegan, C. McCreery and W.T. McNicholas \\ Departments of Respiratory Medicine and Cardiology, and the Respiratory Sleep Laboratory, University \\ College and St Vincent's Hospital, Dublin, Ireland
}

\begin{abstract}
Summary: This report documents how respiratory sleep disorders can adversely effect ischaemic heart disease. Three male patients (aged 60-67 years) with proven ischaemic heart disease are described. They illustrate a spectrum of nocturnal cardiac dysfunction, two with nocturnal angina and one with nocturnal arrhythmias. Full sleep studies were performed in a dedicated sleep laboratory on all patients, and one patient had 48 hours of continuous Holter monitoring. Two patients were found to have obstructive sleep apnoea with apnoea/hypopnoea indices of 57 and 36 per hour, respectively, the former with nocturnal arrhythmias and the latter with nocturnal angina. In both cases, nasal continuous positive airways pressure successfully treated the sleep apnoea, with an associated improvement in nocturnal arrhythmias and angina. The third patient who presented with nocturnal angina, did not demonstrate obstructive sleep apnoea (apnoea/hypopnoea index $=7.2$ ) but had significant oxygen desaturation during rapid eye movement (REM) sleep. This patient responded to a combination of nocturnal oxygen and protriptyline, an agent known to suppresss REM sleep, and had no further nocturnal angina. All patients were considered to be an optimum cardiac medication and successful symptom resolution only occurred with the addition of specific therapy aimed at their sleep-related respiratory problem. We conclude that all patients with nocturnal angina or arrhythmias should have respiratory sleep abnormalities considered in their assessment.
\end{abstract}

\section{Introduction}

Sleep in the normal individual is associated with fluctuations in haemodynamic and respiratory variables, particularly during rapid eye movement (REM) sleep. ${ }^{1}$ However, the role of these changes in the development of nocturnal angina is not well understood. ${ }^{2,3}$ The changes are greatly exaggerated in the presence of respiratory sleep disorders and their potential to adversely affect cardiac function is also increased with coexistent ischaemic heart disease (IHD). ${ }^{4}$ Fluctuations in vagal and sympathetic activity, associated with apnoeic events, may also increase the risk of nocturnal arrhythmias. ${ }^{5}$ We describe below three cases in which a clear association exists between specific sleeprelated respiratory disorders and nocturnal cardiac dysfunction secondary to IHD, and the possible underlying pathophysiological mechanisms are discussed.

Correspondence: W.T. McNicholas, M.D., F.R.C.P.I., Department of Respiratory Medicine, St Vincent's Hospital, Elm Park, Dublin 4, Ireland.

Accepted: 5 October 1993
Methods

All three patients were monitored during normal night time sleep using standard polysomnographic techniques ${ }^{6}$ with continuous recordings of electroencephalography (EEG), electrooculography (EOG) and submental electromyography (EMG). In addition, an electrocardiogram (ECG) was recorded via a single praecordial lead $\left(\mathrm{CM}_{5}\right)$, which allowed accurate heart-rate estimations, but which was not sensitive enough to detect ST segment changes. Respiration was recorded continuously and noninvasively using a respiratory inductance plethysmograph (Respitrace) which was calibrated using the isovolume technique. ${ }^{7}$ When calibrated against a spirometer, this device has been shown by many investigators to provide a relatively accurate means of noninvasively measuring respiration, ${ }^{8,9}$ and thus, is well suited to studying respiration during sleep. The device can also distinguish obstructive from central apnoeas based on the presence or absence respectively of equal and opposite movements in the ribcage and abdomen channels of the respitrace, where an apnoea is indicated by a net tidal volume (sum) of zero lasting at least 10 seconds. Hypopnoea is defined as a period of 10 seconds or more when the tidal volume falls abruptly to less than $50 \%$ of the average tidal 
volume in the preceding 30 seconds, associated with a drop in oxygen saturation of at least $4 \%$ from baseline. The above variables were recorded continuously on a Grass $78 \mathrm{D}$ polygraph recorder. Oxygen saturation was also recorded continuously using an Ohmeda Biox 3700e pulse oximeter. One patient subsequently had 48 hours of continuous Holter monitoring 24 hours after the sleep study. Two of the patients were later admitted for assessment of suitability for nasal continuous positive airways pressure (NCPAP), and one had further Holter monitoring performed while established on NCPAP treatment. The patients' characteristics are summarized in Table I.

\section{Subjects}

Case 1

A 67 year old male was admitted to another institution with an inferior myocardial infarction complicated by sinus bradycardia, first degree heart block and multiple premature atrial complexes. He smoked 20 cigarettes per day and drank 40-60 units of alcohol per week. Overnight oximetry was performed as he was observed to be a heavy snorer and admitted to excessive daytime sleepiness. This showed episodes of marked arterial oxygen desaturation down to $86-64 \%$. During one of these desaturations he developed marked bradycardia and an episode of sinus arrest.

He was referred to our unit for further respiratory sleep assessment. A full sleep study demonstrated severe obstructive sleep apnoea (OSA) with an apnoea/hypopnoea index (AHI) of 57/hour (Figure 1a). Apnoeas were associated with marked oxygen desaturation to levels as low as $51 \%$. Heart rate varied between 40-90/minute. Premature ventricular complexes (PVCs) were noted to occur frequently towards the end of obstructive events. They were particularly common during REM sleep (154/hour), when apnoeas were longer and desaturations more pronounced (Figure 1a), compared to non-REM sleep (9.3/hour). A 48 hour Holter monitor showed multiple arrhythmias during sleep, including multiple PVCs (mean of 45/hour), premature atrial complexes, runs of bigemini and atrial tachycardia, and bradycardia (Figure 2). The only arrhythmias apparent during wakefulness were PVCs. He was commenced on NCPAP at a pressure of $10 \mathrm{~cm} \mathrm{H}_{2} \mathrm{O}$ and sleeprelated desaturations were completely abolished (Figure 1b).

Table I Patient characteristics

\begin{tabular}{|c|c|c|c|}
\hline & Patient 1 & Patient 2 & Patient 3 \\
\hline Age (years) & 67 & 60 & 65 \\
\hline Height (m) & 1.8 & 1.77 & 1.75 \\
\hline Weight (kg) & 112.5 & 90 & 76 \\
\hline $\begin{array}{l}\text { Interval since } \\
\text { myocardial infarct }\end{array}$ & 1 month & 1.5 years & 14 years \\
\hline $\begin{array}{l}\text { Coronary } \\
\text { angiography }\end{array}$ & Not done & Triple vessel disease & Triple vessel disease \\
\hline Nocturnal angina & None & 3-4 episodes/week & $1-2$ episodes/night \\
\hline $\begin{array}{l}\text { Nocturnal } \\
\text { arrhythmias }\end{array}$ & See text & Frequent PVCs & None \\
\hline $\mathrm{PO}_{2}(\mathrm{kPa})$ & 8.8 & 11.5 & 8.2 \\
\hline $\mathrm{PCO}_{2}(\mathrm{kPa})$ & 6.15 & 5.36 & 4.9 \\
\hline $\mathrm{FEV}_{1}(\%$ predicted $)$ & $1.431(43 \%)$ & $3.561(104 \%)$ & $1.201(37 \%)$ \\
\hline FVC (\% predicted) & $2.01(47 \%)$ & $4.231(98 \%)$ & $1.561(38 \%)$ \\
\hline $\mathrm{FEV}_{1} / \mathrm{FVC}$ ratio & $72 \%$ & $84 \%$ & $77 \%$ \\
\hline DLCO (\% predicted) & $69 \%$ & $79 \%$ & Technically impossible \\
\hline $\begin{array}{l}\text { Apnoea hypopnoea } \\
\text { index (per hour) }\end{array}$ & 57 & 36 & 7.2 \\
\hline Lowest $\mathrm{O}_{2}$ saturation & $64 \%$ & $84 \%$ & $83 \%$ \\
\hline $\begin{array}{l}\text { Treatment prior to } \\
\text { sleep assessment } \\
\text { (daily doses) }\end{array}$ & $\begin{array}{c}\text { Isosorbide } \\
\text { mononitrate } 30 \mathrm{mg} \\
\text { Aspirin } 300 \mathrm{mg} \\
\text { GTN as required }\end{array}$ & $\begin{array}{c}\text { Isosorbide } \\
\text { mononitrate } 40 \mathrm{mg} \\
\text { Amlodipine } 10 \mathrm{mg} \\
\text { Bisoprolol } 5 \mathrm{mg} \\
\text { Aspirin } 300 \mathrm{mg} \\
\text { GTN as required }\end{array}$ & $\begin{array}{l}\text { Diltiazem } 180 \mathrm{mg} \\
\text { Glyceryl Trinitrate } 9 \mathrm{mg} \\
\text { Propranolol } 30 \mathrm{mg} \\
\text { GTN as required } \\
\text { I.V. Nitrates PRN }\end{array}$ \\
\hline
\end{tabular}

$\mathrm{PO}_{2}=$ partial pressure of oxygen; $\mathrm{PCO}_{2}=$ partial pressure carbon dioxide; $\mathrm{FEV}_{1}=$ forced expiration volume in the first second; $F V C=$ forced vital capacity; DLCO = transfer factor for carbon monoxide; $P V C=$ premature ventricular complex; GTN = glyceryl trinitrate. 


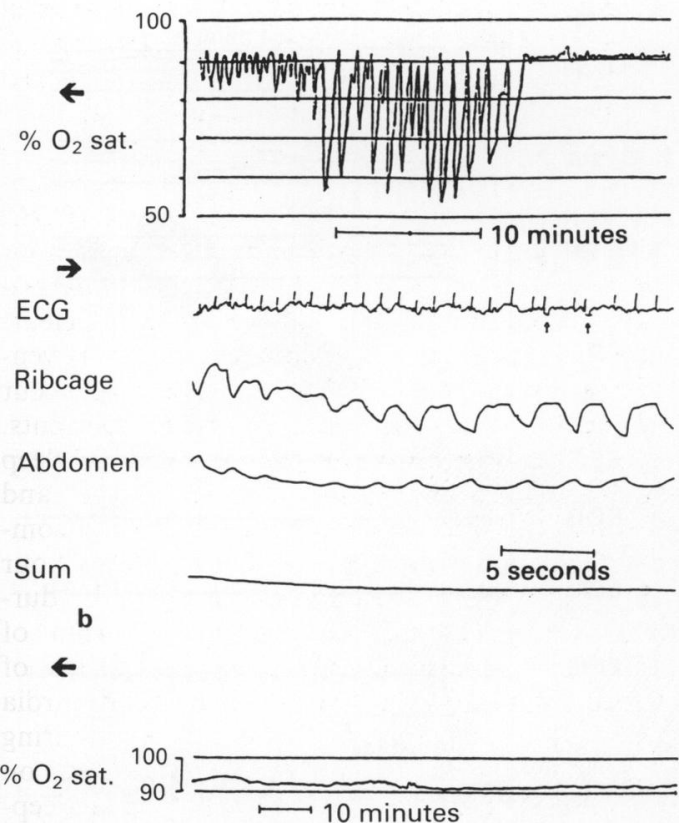

Figure 1 Patient no. 1. (a) An example of his oximetry record during REM sleep showing marked desaturations in association with recurrent obstructive apnoeas. An ECG and repitrace record during a characteristic obstructive apnoea from this period is shown with it. (b) Follow-up oximetry while on $10 \mathrm{~cm} \mathrm{H}_{2} \mathrm{O}$ NCPAP. (Bold arrow denotes direction of recording.)

Continuous strip 02:26 Aug 72 bpm Ch1

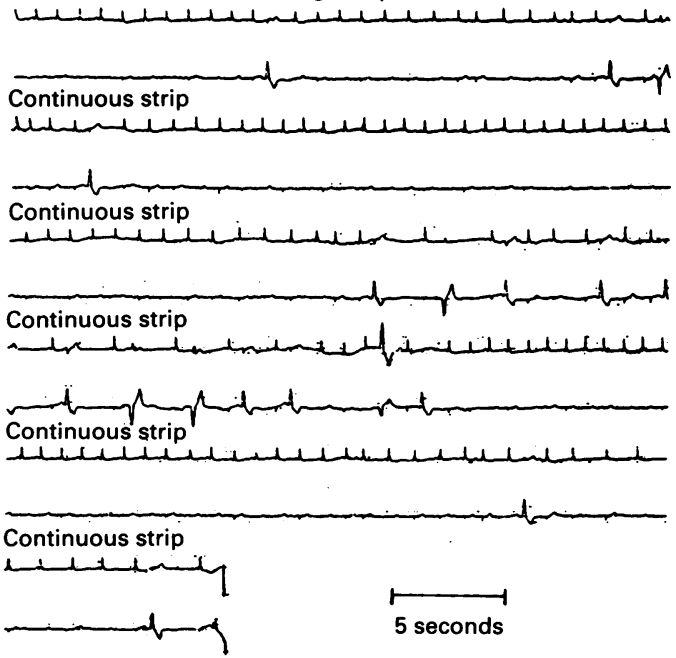

Figure 2 Patient no. 1. Sample printout (02:26 hours) from his $\mathbf{4 8}$ hour Holter monitor displaying fast atrial tachycardia with multifocal ventricular ectopics.
He was discharged home on NCPAP therapy without the need for anti-arrhythmic therapy. On subsequent review, over a 4 month period, the patient reported increased well-being and increasing energy levels. A repeat Holter monitor 2 months later showed a marked reduction in the number of PVCs and absence of any bradycardia, bigemini and atrial tachycardia during sleep.

\section{Case 2}

A 60 year old male was admitted with unstable angina. He had an 18 month history of angina pectoris following a myocardial infarction in 1991 . Coronary angiography showed severe triple vessel coronary artery disease but the patient declined surgery. There was a significant nocturnal component to his problem with wakening from sleep with chest pain 3-4 times per week. His exertional angina settled on increased medication. However, he continued to have nocturnal angina at the same frequency and was thus referred to the sleep laboratory for assessment.

He admitted to habitual snoring, frequent nighttime awakenings and frequent day time naps. $\mathrm{He}$ smoked 10 cigarettes per day and drank 10-15 units of alcohol per week. A formal sleep study showed moderate to severe obstructive sleep apnoea (OSA) with an AHI of 36/hour, which was associated with frequent arterial desaturations occurring at a rate of 31 /hour (Figure 3). He had remained on his antianginal medication during the study and did not awake with chest pain. The ECG lead used during the study was not sensitive enough to detect ST segment changes and thus ischaemic events could not be ruled out. Desaturations were abolished with NCPAP at a pressure of $10 \mathrm{~cm} \mathrm{H}_{2} \mathrm{O}$ during a week's trial in hospital. He had no further nocturnal angina while on NCPAP. He initially declined home NCPAP use and his nocturnal
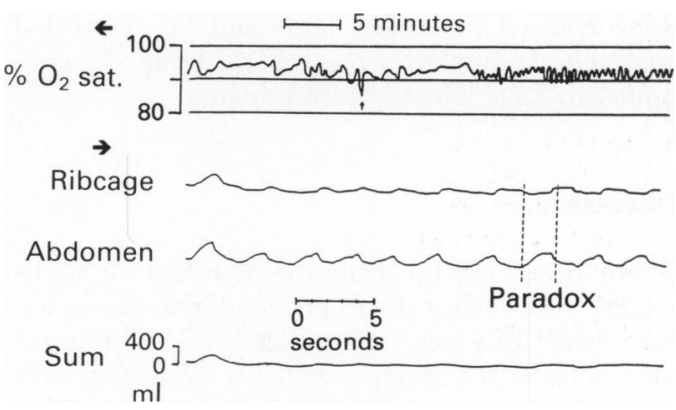

Figure 3 Patient no. 2. Oximetry recording during sleep showing recurrent desaturations. An obstructive apnoea occurring at the time of greatest desaturation $(\uparrow)$ is also shown. 
angina returned with the same frequency. On recommencing NCPAP at home, the frequency of nocturnal angina greatly decreased to about once every 4-6 weeks.

This improvement was not due to impaired arousal due to deeper sleep with NCPAP, since arousal is impaired in patients with sleep apnoea and should improve with successful control.

\section{Case 3}

A 65 year old male had a one year history of angina pectoris following a myocardial infarction in 1978. He had undergone coronary artery bypass grafting and aortic valve replacement in 1983. He was admitted on this occasion with unstable angina with nocturnal angina occurring 2-3 times/night over the previous 2 weeks associated with wakening. He also had congestive cardiac failure and a background of restrictive lung disease which was secondary to asbestosis. He was an ex-smoker and drank 5 units of alcohol per week. His medication was increased in an effort to control his unstable angina and heart failure, but his nocturnal angina failed to improve and he was referred for a full sleep assessment. Overnight sleep study showed an AHI of 7.2/hour, which effectively ruled out any significant OSA (normal range 0-15 apnoeas or hypopnoeas/hour of sleep). He did, however, develop significant arterial desaturation during REM sleep. On two occasions he awoke with his typical anginal pain about 10 minutes after onset of REM sleep, associated with oxygen desaturation and a progressive increase in heart rate (from 75 to $86 /$ minute during the first episode and from 79 to $90 /$ minute during the other). These periods were also marked by the appearance of PVCs (Figure $4 b)$.

He was commenced on nocturnal oxygen supplementation with some improvement in nocturnal angina. After one week protriptyline, a REM suppressing agent, was gradually introduced in an attempt to reduce REM-related desaturations. He was reviewed 3 months later and his nocturnal angina had completely resolved, although he continued to have effort-induced angina.

\section{Discussion}

These three cases illustrate the potential for sleeprelated respiratory disorders to aggravate ischaemic heart disease. Two patients had significant obstructive sleep apnoea, which is associated with marked swings in intrathoracic pressure resulting from repetitive inspiratory efforts against an occluded upper airway (Mueller manoeuvre). During the early part of an obstructive apnoea there is intense vagal stimulation with resulting bradycar-
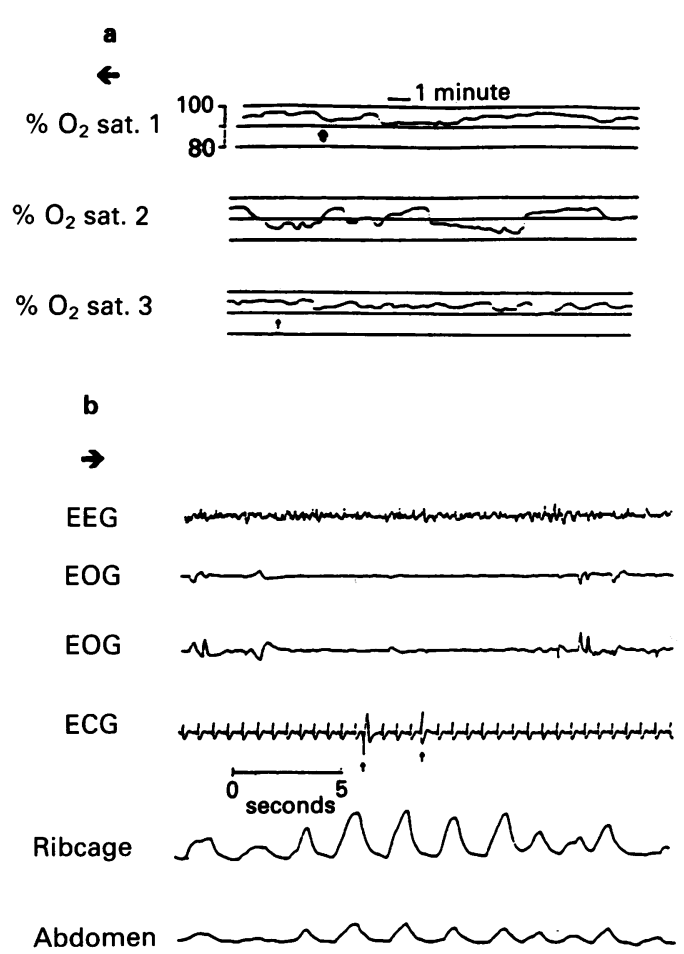

Sum

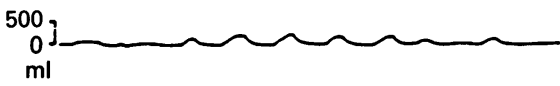

Figure 4 Patient no 3. (a) Three episodes of desaturation associated with REM periods. The first and third episodes were marked by the patient waking with angina (indicated by arrows, $\uparrow$ ). He had taken sublingual glyceryl trinitrate 15 minutes before the second episode. (b) This demonstrates the abrupt falls in ribcage contribution to breathing with rapid eye movements (EOG channels) leading to reduction in tidal volume (sum) and oxygen desaturation as in (a). Associated multifocal ventricular ectopics are indicated in the ECG record.

dia and a fall in blood pressure. This is then followed by increased sympathetic stimulation secondary to the fall in oxygen saturation that is characteristic of the apnoea, with associated increases in heart rate and blood pressure. ${ }^{10}$ These cyclical changes lead to marked sinus arrhythmia, which is typical of OSA. ${ }^{11}$ Frequent ventricular ectopics are also described in association with OSA. ${ }^{12}$ In a patient who is already susceptible to arrhythmias, i.e. post-myocardial infarction, these changes can lead to serious arrhythmias as illustrated by case 1 above. Tilkian et al. have previously reported arrhythmias in association with OSA and their abolition following tracheostomy. ${ }^{5}$ 
There have been several studies attempting to document the underlying mechanisms leading to nocturnal angina. Nowlin and coworkers reported that the majority of episodes of nocturnal angina were associated with REM sleep..$^{13}$ Other authors have emphasized the importance of impaired left ventricular function secondary to recumbency, ${ }^{14}$ increased heart rate, ${ }^{3}$ diurnal variation of coronary artery tone, ${ }^{15}$ or arousals and body movements. ${ }^{2}$ It is likely, however, that in any given patient the pathogenesis is multifactorial.

The relationship between nocturnal angina and OSA is not well understood, and there is some evidence that OSA may even be a risk factor for the development of ischaemic heart disease. ${ }^{16}$ Factors that could impair cardiac function include a reduction in ejection fraction caused by the Mueller manoeuvre, ${ }^{17}$ swings in blood pressure and heart rate, and repeated arousals. ${ }^{10}$ However, one report failed to show any immediate ill effects due to disordered breathing and hypoxia in a group of patients with IHD. ${ }^{8}$

The cardiorespiratory changes that occur during sleep are not uniform. ${ }^{10}$ During non-REM sleep there is a decrease in mean arterial pressure of between 5 and $6 \mathrm{~mm} \mathrm{Hg}$, a decrease in cardiac output of $0-10 \%$ and a decrease in heart rate of between 5 and $10 \%$. In contrast REM sleep is a time of major autonomic arousal and there are significant fluctuations in the above parameters. There is also a physiological decrease in alveolar ventilation during sleep, which is associated with only minor falls in arterial $\mathrm{PO}_{2}$ in normal subjects, ${ }^{19}$ but may result in major desaturations in patients with severe lung disease especially chronic obstructive pulmonary disease. ${ }^{20}$ The potential effect of these changes on cardiopulmonary performance is illustrated in case 3 above. Both his restrictive lung disease and congestive cardiac failure would have tended to reduce arterial oxygenation. He was able to partially compensate for this during wakefulness by hyperventilating. However, this tended to be offset by the normal sleep-related reductions in ventilation, particularly during phasic REM sleep, when there is a significant reduction in the ribcage contribution to breathing. This reduction in ventilatior resulted in significant oxygen desaturation and increase in heart rate both of which contributed to his angina. Protriptyline, a tricyclic antidepressant that suppresses REM sleep, ${ }^{21}$ abolished his nocturnal angina. While oxygen desaturation during REM sleep was likely to be a major factor in this patient's nocturnal angina, the autonomic changes associated with REM may also have contributed.

We conclude that in patients with predominantly nocturnal cardiac dysfunction, the possibility of an underlying respiratory sleep disorder should be considered. In addition to clinical assessment, simple overnight oximetry recordings may provide important diagnostic clues in the evaluation of such patients.

\section{Acknowledgements}

We would like to acknowledge Dr P. Quigley and Dr S. O'Neill for referring the patients in this study.

\section{References}

1. Somers, V.K., Dyken, M.E., Mark, A.L. \& Abboud, F.M. Sympathetic-nerve activity during sleep in normal subjects. $N$ Engl J Med 1993, 328: 303-307.

2. Quyyumi, A.A., Efthimiou, J., Quyyumi, A., Mockus, L.J., Spiro, S.G. \& Fox, K.M. Nocturnal angina: precipitating factors in patients with coronary artery disease and those with variant angina. $B r$ Heart $J$ 1986, 56: 346-352.

3. Quyyumi, A.A., Wright, C.A., Mockus, L.J. \& Fox, K.M. Mechanisms of nocturnal angina pectoris: importance of increased myocardial oxygen demand in patients with severe coronary artery disease. Lancet 1984, i: 1207-1209.

4. Partinen, M., Putkonen, P., Kaprio, J., Koskenvuo, M. \& Hilakivi, I. Sleep disorders in relation to coronary heart disease. Acta Med Scand 1982, 660: 69-83.

5. Tilkian, A.G., Guilleminault, C., Schroeder, J.S., Lehrman, K.L., Simmons, F.B. \& Dement, W.C. Sleep induced apnoea syndrome: prevalence of cardiac arrhythmias and their reversal after tracheostomy. Am J Med 1977, 63: 348-358.

6. Rechtschaffen, A. \& Kales, A. A Manual of Standardised Terminology, Techniques and Scoring System for Sleep States of Human Subjects. National Institutes of Health, Washington, D.C., 1968 (publication no. 204).

7. Konno, K. \& Mead, J. Measurement of the separate volume changes of ribcage and abdomen during breathing. $J$ Appl Physiol 1967, 22: 407-422.
8. Zimmerman, P.V., Connellan, S.J., Middleton, H.C Tabona, M.V., Goldman, M.D. \& Pride, N. Postural changes in ribcage and abdominal volume-motion coefficients and their effect on the calibration of a respiratory inductance plethysmograph. Am Rev Respir Dis 1983, 127: 209-214.

9. Hudgel, D.W., Capehart, M., Johnson, B., Hill, P. \& Robertson, D. Accuracy of tidal volume, lung volume and flow measurements by inductance vest in COPD patients. $J$ Appl Physiol 1984, 56: 1659-1665.

10. Shepard, J.W. Cardiopulmonary consequences of obstructive sleep apnoea. Mayo Clin Proc 1990, 65: 1250-1259.

11. Guilleminault, C., Connolly, S., Winkle, R., Melvin, K. \& Tilkian, A. Cyclical variation of the heart rate in sleep apnoea syndrome: mechanisms and usefulness of $24 \mathrm{~h}$ electrocardiography as a screening technique. Lancet 1984, 1: 126-131.

12. Shepard, J.W., Garrison, M.W., Grither, D.A. \& Dolan, G.F Relationship of ventricular ectopy to oxyhemoglobin desaturation in patients with obstructive sleep apnoea. Chest 1985, 88: $335-340$.

13. Nowlin, J.B., Troyer, W.G., Collins, W.S. et al. The association of nocturnal angina with dreaming. Ann Intern Med 1965, 63: 1040-1046.

14. Gorlin, R. Pathophysiology of cardiac pain. Circulation 1965 , 32: $138-148$. 
15. Waters, D., Miller, D., Bouchard, A., Bosch, X. \& Theroux, P. Circadian variation in variant angina. Am J Cardiol 1984, 54: 61-64.

16. Hung, J., Whitford, E.G., Parsons, R.W. \& Hillman, D.R. Association of sleep apnoea with myocardial infarction in men. Lancet 1990, 336: 261-264.

17. Scharf, S.M., O'Bierne Woods, B., Brown, R., Parisi, A., Miller, M.M. \& Tow, D.E. Effects of the mueller manoeuvre on global and regional left ventricular function in angina pectoris with and without previous myocardial infarction. Am J Cardiol 1987, 59: 1305-1309.

18. De Olazabal, J.R., Miller, M.J., Cook, W.R. \& Mithoefer, J.C. Disordered breathing and hypoxia during sleep in coronary artery disease. Chest 1982, 82: 548-552.
19. Block, A.J., Boysen, P.G., Wynne, J.W. \& Hunt, L.A. Sleep apnea, hypopnea and oxygen desaturation in normal subjects: a strong male predominance. $N$ Engl J Med 1979, 300: 513-517.

20. Douglas, N.J., Calverley, P.M.A., Leggett, R.J.E., Brash, H.M., Flenley, D.C. \& Brezinova, V. Transient hypoxaemia during sleep in chronic bronchitis and emphysema. Lancet 1979, i: $1-4$.

21. Brownell, L.G., West, P., Sweatman, P., Acres, J.C. \& Kryger, M.H. Protriptyline in obstructive sleep apnea, a double blind trial. $N$ Engl J Med 1982, 307: 1037-1042. 